\title{
Individual Aesthetic Differences Evaluation of Yan'an Urban Forests in the Loess Plateau China
}

\author{
Mingxia Zhang ${ }^{1}$, Dexiang Wang ${ }^{1 *}$, Zhitao Wang ${ }^{2}$, Xinping Zhang ${ }^{1}$, Ganggang \\ Zhang $^{1}$ and Yong $\mathrm{Xu}^{1}$ \\ ${ }^{1}$ Northwest A\&F University, Yangling 712100, China \\ ${ }^{2}$ Qinghai Academy of Agriculture and Forestry Sciences, Xining 810016, China
}

\section{Abstract}

Urban forests have important aesthetic values contributing to the quality of urban life. The aesthetic difference in urban forests can cause negative or positive externalities, e.g., the loss or increase of non-priced benefits. Urban forestry planning, therefore, should systematically assess and commensurately measure the aesthetic valye of urban forests, i.e., in monetary terms with material values. Here werpresent an empirical study for assessing the aesthetic value of urban forests using on-site survey datafrom 1,000 visitors in the loess hilly-gully area of Loess Plateau (Yan'an, northern Shaanxi, China). An assessment model is established by logistic regression and automated neural networks for measuring the objective and subjective influencing factors of aesthetic attitude and individual willingness to pay (WTP) recreation fees for consumption of the urban forest environment. The objective factors are directly linked to WTP and gains the largest weight in the system; The subjective factors exert strong influences on WTP; additionally, quality of urban virescence strongly affects the respondents' aesthetic attudes, thus'influencing their WTP. Our results are useful for assessing ecosystem sernice function in urban forests, thus contributing to the management and sustainable development of prban forests in the study area as well as other forests in similar setting.

Keywords: Loess Plateau. yan'an; Logistic regression ; Automated neural networks

\section{Introduction}

Aesthetic is the center of everything that is considered valuable by human beings, and individual differences in aesthetic attitude affect the aesthetic value [1-2]. In forest planning, aesthetic value is an important criterion for assessing the quality of urban forests. Being an aesthetic object, urban forests are a group of public goods whose aesthetic value refers to the individuar willingness to pay (WTP) recreation fees for consumption of the pleasant urban forest environment [3-4]. In social psychology, WTP is considered to be the behavioral intention that affects the attitude partially and helps to predict behavior [5-6]. The aesthetic value of urban forests has an indeterminate price but lacks a market price [7]. Therefore, policy makers are required to carry out direct monetary assessment on the aesthetic value of urban forests, similar as the work for valuation of other ecosystem service functions. In this way, the aesthetic value of urban forests will gain comparability with economic factors [1].

In the loess hilly-gully area on China's Loess Plateau, urban forests play a special and important role in the land area and the life of residents. These forests possess regional, temporal, integral, and participatory features because of the unique geological and cultural backgrounds. The city of Yan'an in northern Shaanxi Province is regarded as the cultural, economic, tourism, and transportation center of the loess hilly-gully area. In particular, 
Yan'an urban forests are the witness of China's revolutionary history and the symbol of Yan'an spirit, thus have gained great attention from the government and publics. Urban forestry construction in Yan'an is of great significance to the establishment of a garden city and an ecological city in the loess hilly-gully area.

Here we present a one-year empirical study on urban forests at the revolutionary site of Yan'an, which represent vast forest areas on the Loess Plateau. The aesthetic value of urban forests was assessed by using CVM and the influencing factors of individual WTP and aesthetic differences were identified by logistic regression analysis and automated neural networks. The results will provide reference data for assessing the quality of urban forest ecosystem service function, further contributing to the management and sastainable development of urban forests.

\section{Study Area Description}

Site survey data were collected from urban forests in Yan'an city dorthern Shaanxi Province, China, part of the loess hilly-gully area of Loess Plateau (Figure1). The loess hillygully area of Loess Plateau is a typical hilly reg on of loess fidges and hills, where land surface is highly fragmented and soil erosion widely oecurs. This area is deeply cut by gullies with steep slopes. This topography has limited the expansion of townships, so that the urban area is relatively small and large-area suburban landscaping has become one of the main tasks for constructing an urban ecological netwerk.

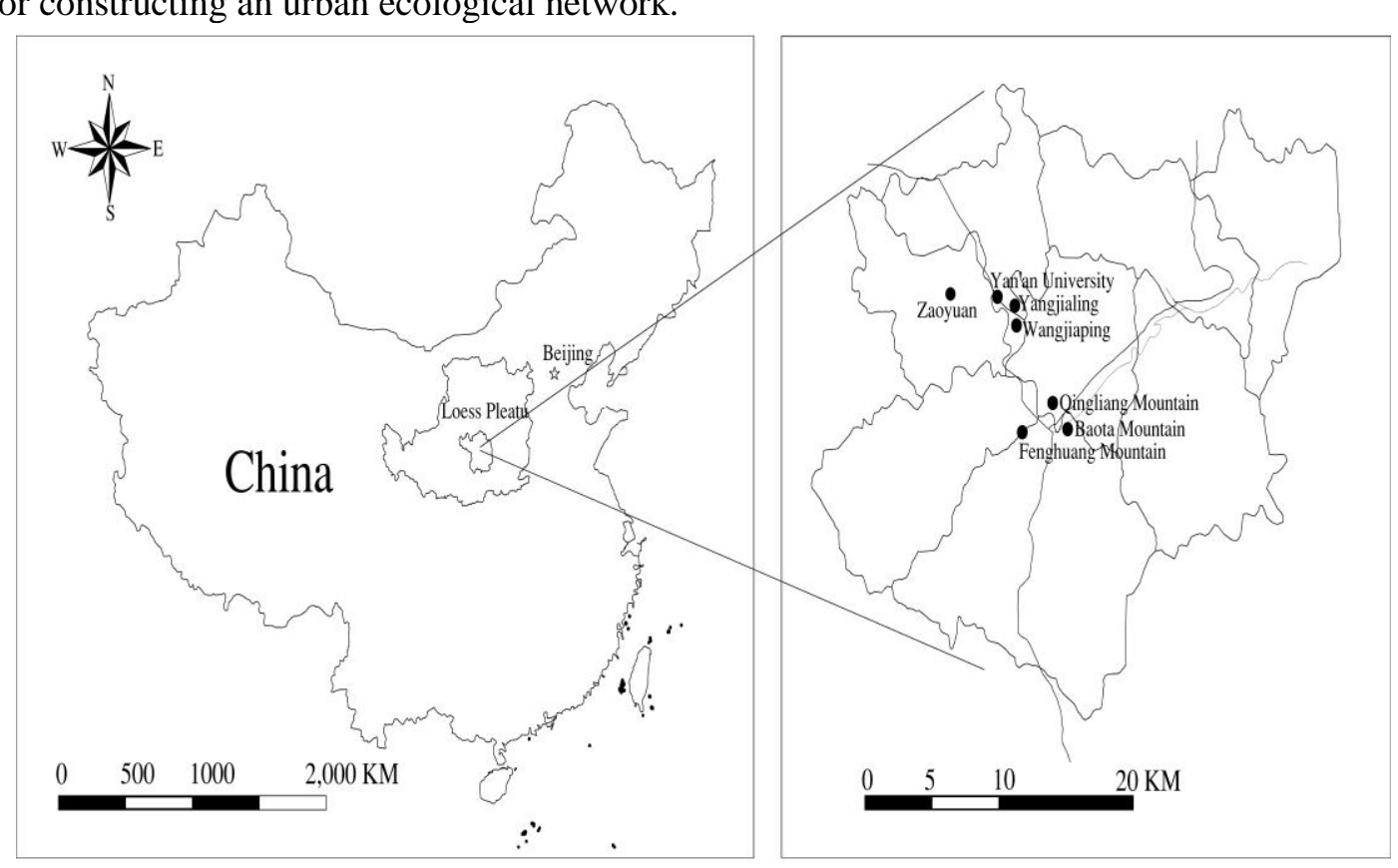

Figure 1. Location of the Study Area in Yan'an, Shaanxi Province, China

The study area experiences a continental monsoon climate. Average annual change rate of precipitation is $20 \sim 30 \%$, and the precipitation is 3 5-time greater in rainy years than in rainless years (up to 10-time greater in some areas) [16]. In 1999, the Yan'an government proposed an overall design of urban forest ecosystem based on the Grain for Green Project. Major types of the existing urban forests include road green space, scenic forest, memorial green space, square garden, exclusive green space, street trees, and suburban shelterbelt. 
Regarding the natural distribution of vegetation, the study area lies in a warm-temperate semi-arid forest-steppe hydraulic erosion zone associated with drought, lack of soil moisture, and evapotranspiration far greater than precipitation. In history, forest and grass thrived in northern Shaanxi with picturesque scenery before the Qin and Han dynasties (even Tang dynasty). Since the Tang and Song dynasties, however, forest vegetation had been diminished with intense deforestation and grassland destruction. Taking as an example the county of Yanchuan, the remaining forest area accounted for $26 \%$ of the total arable land area in 1949, and the ratio of forest area declined to $7.3 \%$ in 2000 , with $19 \%$ forest area cleared for food production.

In 1999, the Yan'an government proposed an overall design of urban forest écosystem based on the Grain for Green Project. Major types of the existing urban forests include road green space, scenic forest, memorial green space, square garden, exclusive green space, street trees, and suburban shelterbelt.

Scenic forests are mainly distributed in the mountains of Babaơ, Fenghuang, and Qingliang. In these areas, virescence has never been interrupted from the Yan'an revolutionary period until now. Associated forest management and improvement are strict and meticulous, thereby forming the present scenic forests in a neat form with good growth of trees and a high diversity of plant species.

Due to limited supply of land, exclusive green spaces of government agency, enterprises, and institutions cover small areas, mainly in schools and collages. These areas, as represented by the campuses of Yan'an University @and Yan'an Irormal School, are subjected to less destruction by the construction of municipal works. Together with continuous selection and improvement for years has contributed to the present stable phase of forest ecosystem service functions such as green landscape effect and humanistic spirit expression.

Memorial green spaces take a special position in the politics and culture of Yan'an. During the revolutionary period, leaders of the Central Committee of the Communist Party (CPC) of China lived in Yan'an and virescence by tree planting was begun around their living space. These memorial green spaces had become places of sightseeing since the 1950s to 1960s, which received a total of 5.594 million of visitors during January-July 2010. At present, the memorial green spaces tend 48 be scientific and rational in terms of selection of tree species, configuration, and layout of small landscape patches.

The established shenerbelts were artificially planted in the 1960 s to 1970 s, mainly including Robinia pseudoacacia (>90\%). Presently, alternative tree species with superior comprehensive traits tor adaptability, resistance, competitiveness, and growth potential are in need, and restotation and improvement techniques are applied for degraded $R$. pseudoacacia forests.

Road green spaces and square gardens have been built over the past 10 years. These are mainly lawn or grass savanna dominated by exotic plants. To ensure their normal growth, artificial irrigation is carried out 3-5 times in rainless years. This strategy has increased the management and protection cost and reduced the resource utilization efficiency in Yan'an where there has been a shortage of water resources. Although native trees and shrubs such as Pinus tabulaeformis and Syringa spp. have been supplemented in some areas, the management cost remains high and the ecosystem services is relatively weak.

\section{Material and Methods}

Aesthetic attitude has been considered statistically significant in a variety of CVM-based modeling studies [17]. A few studies have established CVM models by considering both individual WTP and altitude toward recreation fees [18]. In the present study, we assess the 
visitors' WTP for urban forest recreation environment more specifically by including both objective and subjective influencing factors of aesthetic attitude.

\subsection{Data Collection and Field Survey}

The year of 2011 was the ninetieth anniversary of the Communist Party of China foundation and turned to be a national red tourism year in China. During July 2011 to August 2013, numerous tourists visited the Yan'an Revolutionary Base Area. Thus, a one-year site survey was conducted by questionnaire for visitors to urban forests in Yan'an. The questionnaire comprised three sections (Table 1): 1) Personal information of the respondents; 2) Core valuation by inquiring whether the respondents are desired to consume for sustainable existence of tourism resources in Yan'an scenic areas (including the culture, art, and scenery) and willing to pay a certain amount of protective fee, in order to distinguish negative (0) and positive WTP (1). When the respondents chose "Yes", he or she was asked to list the range of amount willing to pay; when the respondents chose "No" he or she was asked to list the reason for unwilling to pay; 3) Suggestions and recommendations for the government and urban forest development.

The sampling date (2-3 d per week), time, and site were chosen using a hierarchical random sampling design. The sampling time was chosen at $2 \mathrm{~h}$ after sunrise or $1.5 \mathrm{~h}$ before sunset, according to the recreation time of most respondents. The sampling sites (Figure 1) included seven representative zones of Yan an urban forests: Baota Mountain, Qingliang Mountain, Fenghuang Mountain, Wangjaping, Zabydan, Yangjialing, and the urban periphery of Yan'an University. An attempt was made to interview every visitor, and each person can only complete one questionnaire. A total of 1,000 visitors were surveyed, of which 947 completed the questionnaire.

The respondents are moftly Chinese with few foreigners from other countries. Local visitors are from Yan'an and Shaanxi Province respectively account for 58.3\% and $15.9 \%$ of the respondents, followed by those from North China (10.8\%). Other visitors are from Northeast, South, Central, Southryest, and Northwest China. Local visitors from Yan'an commonly choose to take buses $(34.8 \%)$ or walk $(17.7 \%)$, of which $59.1 \%$ have visited urban forests for more than 3 times and mostly have one-off consumption < $200 \mathrm{CNY}$. Non-local visitors from Shaanxi Provirce commonly choose to drive $(29.0 \%)$, of which $10.4 \%$ have visited the forests twice and mostly have one-off consumption of 200-500 CNY each. Visitors from other(provinces commonly choose to take coach (17.9\%), of which $30.5 \%$ have visited urban forests once and mostly have relatively high one-off consumption.

Most visitors consider that the traffic condition should be improved; the dining and drinking, lodging, and public security are satisfactory, but the sanitation is not; additionally, the communication and network operation are satisfactory. Nearly half of the visitors consider that their main purposes are achieved (54.7\%), and that urban virescence is implemented well in Yan'an (56\%). Suggestions for improving urban forests were mainly related to the strengthening of ecological benefits and the increase in urban forest construction, which aim to further improve the quality of virescence and enhance the strength of advertisement for attracting more visitors. 


\section{Table 1. Definition of Variables and Sample Distribution in the Questionnaire} for Visitors in Yan'an Urban Forests

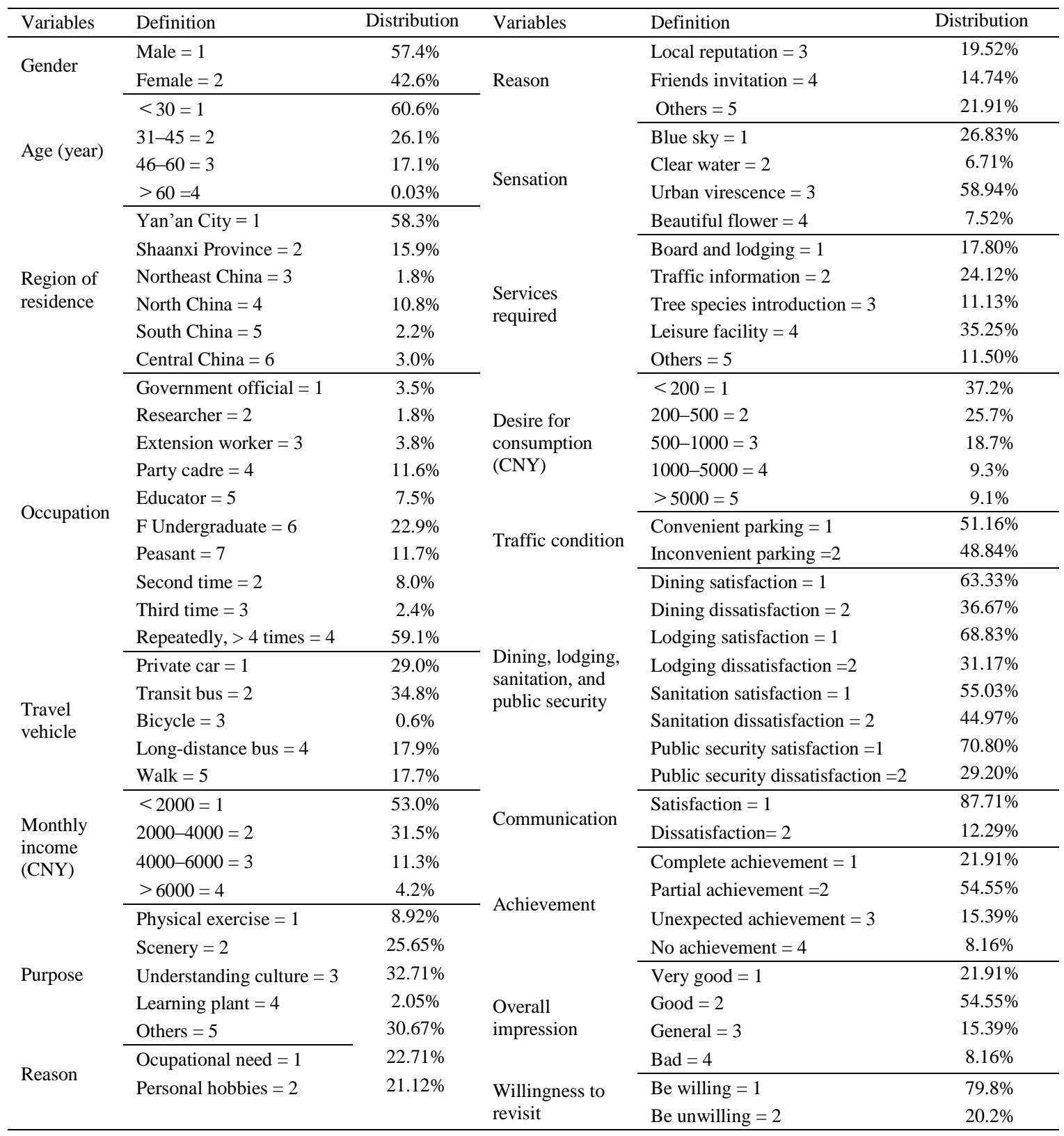

\subsection{Logistic Regression Model}

In the proposed model, the dependent variable is such a binary discrete variable $(1,0)$ can not be directly analyzed by ordinary method of multiple linear regression analysis. Here we select a binary logistic regression model for qualitative variables as shown below:

$$
p_{i}=F\left(\alpha+\sum_{j=1}^{n} \beta_{j} x_{i j}\right)=1 /\left\{1+\exp \left[-\left(\alpha+\sum_{j=1}^{n} \beta_{j} x_{i j}\right)\right]\right\}
$$


A linear expression of this model is obtained by taking logarithm of Formula (1):

$$
\ln \left(\frac{p_{i}}{1-p_{i}}\right)=\alpha+\sum_{j=1}^{n} \beta{ }_{j} x_{i j}+\mu
$$

In Formula (1) and (2), ${ }^{p^{i}}$ is the probability of visitor ${ }^{i}$ for paying aesthetic value of urban forests; ${ }^{x_{i j}}\left({ }^{j}=1,2,3, \ldots \ldots,{ }^{n}\right)$ is the variable ${ }^{j}$ that affects the WTP of visitor ${ }^{i}\left({ }^{n}\right.$ is the number of independent variables); ${ }^{\mu}$ is the random disturbance term; ${ }^{\beta}{ }^{0}$ is a constant; and ${ }^{\beta}{ }_{j}\left({ }^{j}=1,2,3, \ldots \ldots,{ }^{n}\right)$ is the regression coefficient of an independent variable, which can be obtained by maximum likelihood estimation.

\subsection{Automated Neural Networks Method}

Neural networks have a remarkable ability to derive and extract meaning, nutes and trend from complicated, noisy and imprecise data. The neuron receives signals from many sources. The output of the neuron is actually predictions of the single neuron model for a variable in the data set which is referred to as the target.

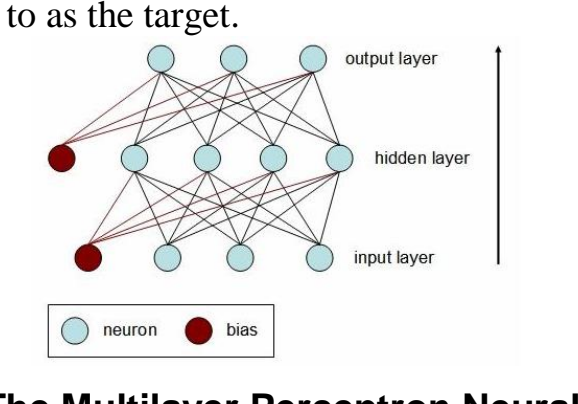

\section{Figure 2 The MultilayerPerceptron Neural Network}

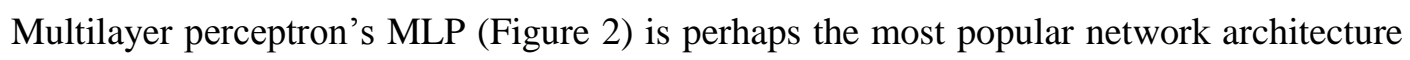
in use today. The neurons and biases are arranged in a layered feed forward topology. The network thus has a simple interpretation as a form of input-output model, with the weights and thresholds as the adjustable parameters of the model. Important issues in Multilayer Perceptron's design includespecification of the number of hidden layers and the number of units in these layers [8-9]. Others include the choice of activation functions and methods of training.

\section{Analysis on' Influencing Factors}

\subsection{Inflencing Factors of Urban Forest Visitors}

A 5-point scale for the strength and direction of individual WTP was used to classify the one-off consumption of visitors into five levels: low (<200 CNY), medium (200-500 CNY), relatively high (500-1,000 CNY), high (1,000 CNY), extremely high (>5,000 CNY). Based on the above classification, the survey covered the visitors' basic characteristics (objective factors) as well as consumer behaviors and aesthetic evaluation (subjective factors) in urban forests (Table 2).

Table 2. Regression Summary for Influencing Factors on Urban Forest Aesthetics Values

\begin{tabular}{cccccc}
\hline No. & variable & BETA & St. Err. of BETA & Partial Cor. & p-level \\
\hline 1 & Sites & 1.250919 & 0.185915 & 0.216007 & $0.000000^{*}$ \\
2 & Gender & 0.076702 & 0.042201 & 0.059653 & 0.069460
\end{tabular}




\begin{tabular}{cccccc}
3 & Age & 0.101800 & 0.031196 & 0.106682 & $0.001142^{*}$ \\
4 & Region & 0.117694 & 0.034302 & 0.112102 & $0.000628^{*}$ \\
5 & Occupation & 0.282348 & 0.100073 & 0.092371 & $0.004884^{*}$ \\
6 & Time & 0.150223 & 0.088629 & 0.055644 & 0.090419 \\
7 & Traveling & 0.350944 & 0.120317 & 0.095467 & $0.003622^{*}$ \\
8 & Monthly income & 0.153608 & 0.058745 & 0.085659 & $0.009072^{*}$ \\
9 & Purpose & 0.121897 & 0.037040 & 0.107578 & $0.001036^{*}$ \\
10 & Reason & 0.142880 & 0.043171 & 0.108182 & $0.000970^{*}$ \\
11 & Sensation & 0.170294 & 0.070949 & 0.078675 & $0.016581^{*}$ \\
12 & Services required & 0.061119 & 0.051171 & 0.039241 & 0.232631 \\
13 & Willingness to increase price & 0.006603 & 0.034041 & 0.006378 & 0.846229 \\
14 & Traffic condition & 0.077205 & 0.044548 & 0.056891 & 0.083414 \\
15 & Parking & 0.018850 & 0.042963 & 0.014425 & 0.660941 \\
16 & Dining & 0.000769 & 0.039824 & 0.000635 & 0.984589 \\
17 & Lodging & 0.029114 & 0.048590 & 0.019697 & 0.549200 \\
18 & Sanitation & 0.029835 & 0.042570 & 0.023037 & 0.483585 \\
19 & Public security & 0.046156 & 0.048879 & 0.031033 & 0.345266 \\
20 & Communication & 0.090177 & 0.031324 & 0.094233 & $0.004084^{*}$ \\
21 & Achievement & 0.071391 & 0.053327 & 0.043975 & 0.180986 \\
22 & Overall impression & 0.069728 & 0.031410 & 0.072798 & $0.026663^{*}$ \\
\hline p $<0.05$. & & & 0 & 0
\end{tabular}

During the onsite survey, we found that the man causes for aesthetic differences include objective factors (gender, age, and monthly income) as well as subjective factors (purpose, reason, and sensation). Difference in the aesthetie attitude-affects individual WTP, which refers to the individual attitude and expeeted behavior toward the payment for the aesthetic value of urban forests. In the theory of rational and planned behavior, the strength of behavioral intentions indicates the extent to which individuals are willing to perform the acts. In CVM, however, the WTP specified may nor reflect any strength of individual WTP. Therefore, the respondents were inquired in the attitude measurement to what extent they would support or oppose a non-mandatory individual WTP.

Results show that among all the influencing factors, sites gains the largest partial correlation coefficient with the amount ofconsumption (0.216007), followed by the region of residence $(0.112102)$, reason for visits $(0.108182)$, purpose of visits $(0.107578)$, and age $(0.106682$, Table 1$)$. These results demonstrate that site is the most important factor affecting the touriss's WTP wile age, region of residence, reason for visits, and purpose of visits pose influences to lesser degrees.

\subsection{Analysis on Influences of Objective Factors}

As shown in Figure 3-1, Figure 3-2, Figure 3-3 and Figure 3-4, the objective factors (tourists Eigen means of traveling, place of residence, monthly income) are directly linked to WTP. This result indicates that the older the age of visitors, the high the possibility of unwilling to pay. Visitors from closer areas are more unwilling to pay. Travel tool selection has no ignificant influence on WTP, despite it reflects the travel distance or personal preference. This result demonstrates that monthly income has a direct and significant influence on WTP. Respondents with high monthly income are more likely to pay. 
International Journal of Multimedia and Ubiquitous Engineering
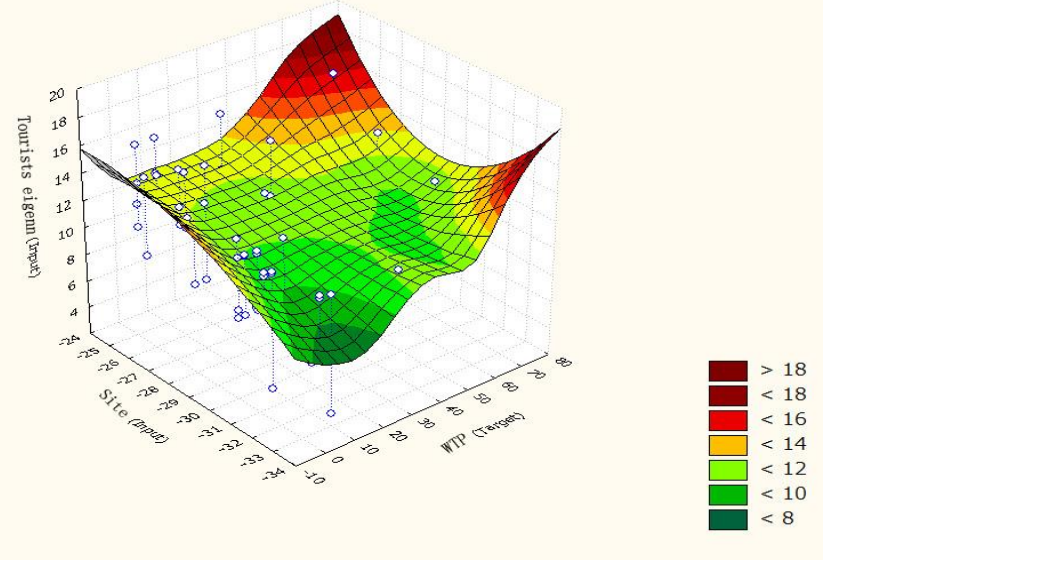

Figure 3-1. The Tourists Eigen of Yan an Urban Forest

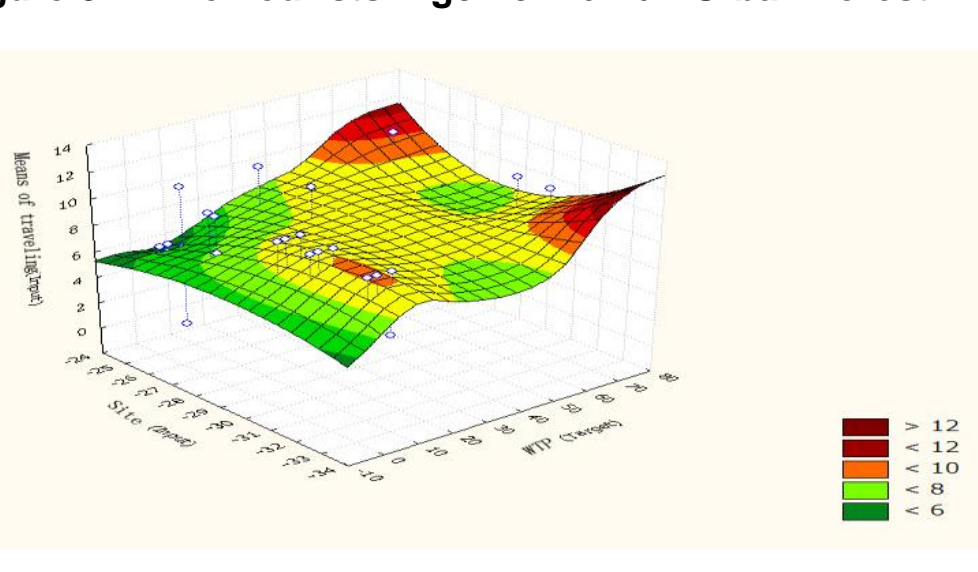

Figure 3-2. The Means of Traveling of Yan'an Urban Forest

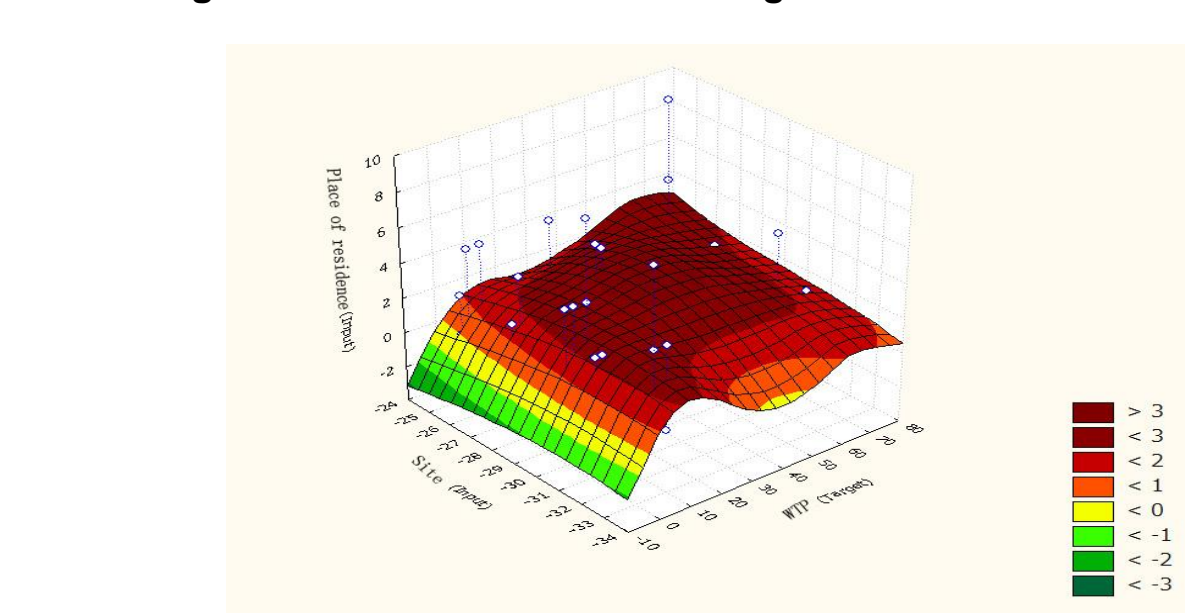

Figure 3-3. The Place of Residence of Tourists of Yan'an Urban Forest 

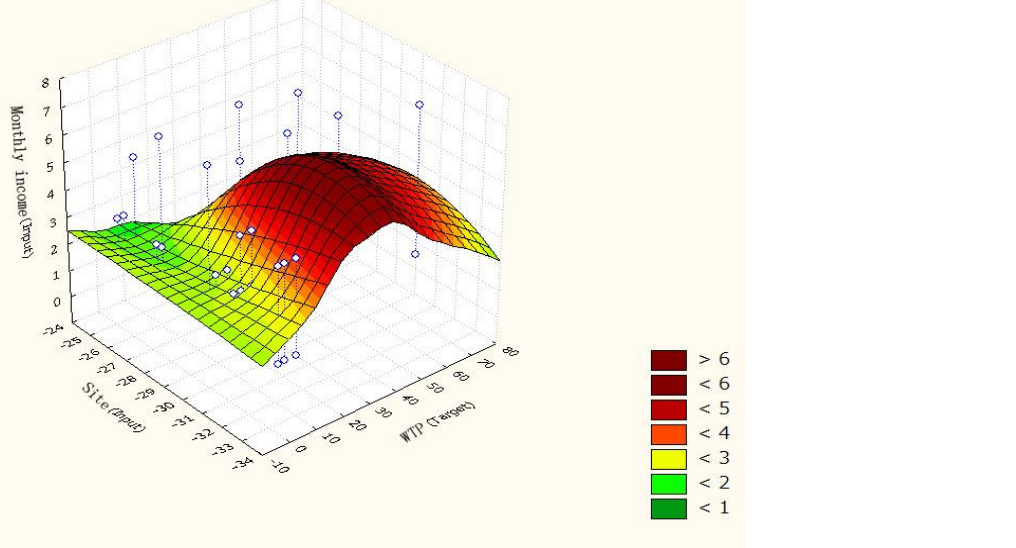

Figure 3-4. The Monthly Income of Tourists of Yan'an Urban Forest

\subsection{Analysis on Influences of Subjective Factors}

The subjective factors (purpose demand, the urban environment impression, sensory evaluation) exert strong influences on WTP.

According to Figure 3-5, among different purposes of the visit to urban forests, enjoy-thebeautiful-scenery gains the largest regression coefficient That is, the purpose of visit has a significant influence on WTP.

Through Figure 3-6, it can be observed that the urban environment impression influence of the respondents' experience, quality of urbacgreening shows the greatest influence. Thus, green quality directly affects the visitors' experiences and the better the green quality, the lower the possibility, of negative attitude toward paying for urban forest recreation environment.

Seeing Figure 3-7, among different reasons for visiting urban forests, work-related reason gains the largest regyession coefficient. This shows that various reasons for visiting urban forests result in significant changes in the respondents' WTP.

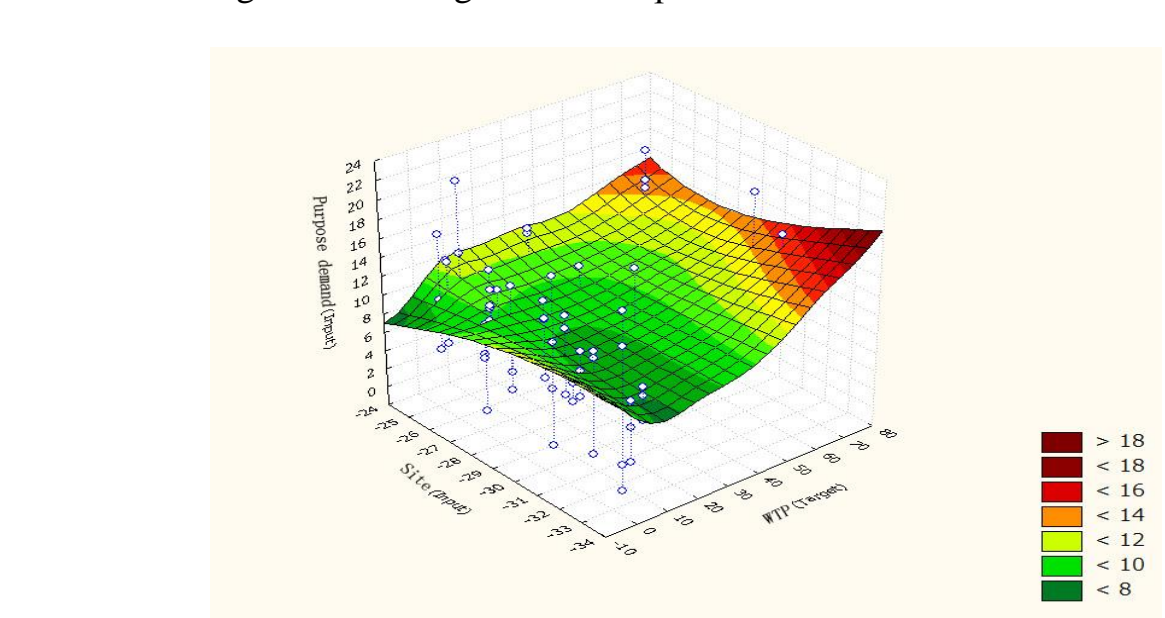

Figure 3-5. The Purpose Demand of Yan'an Urban Forest 


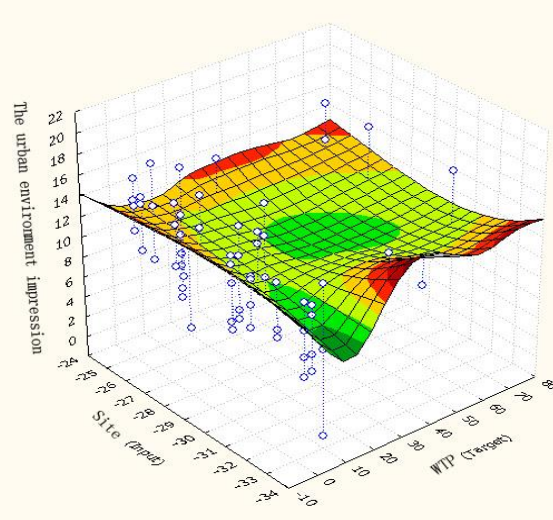

Figure 3-6. The Purpose Demand of Yan'an Urban Forest

Figure 3-7. The Sensory Evaluation of Tourists of Yan'an Urban Forest

\section{Discussion andConclusions}

Our assessment results show that aesthetic differences in individual respondents are statistically signuificant in terms of objective (age and monthly income) and subjective factors (visiting purpose, visiting reason, and personal sensation of urban virescence). Objective factors directly affect WTP and gain the greatest weight in the assessment system, whereas subjective factors as well have profound influences on WTP, consistent with previous findings [10-12]. Specifically, negative aesthetic attitude is related to WTP declines while positive aesthetic attitude is related to WTP increases. Additionally, WTP shows a declining tendency with increasing age, and visitors with the purpose for scenery or work are more likely to pay.

Aesthetics of urban forests is not the ultimate goal, but is a consequence of urban forest construction. Urban forest construction from a single perspective of scenery is not advantageous, but a combination of cultural and historical factors can transform the aesthetic attitude. Extensive studies have shown that the aesthetic attitude can be changed through education and persuasive, targeted informative activities [13-15]. In order to increase the visitors' WTP in the premise of complying with the charging policy, forest managers and policy makers can consider information transmission to visitors with negative aesthetic 
attitudes through education, namely, informing them of the reasons for charges and related policies. For future assessment and prediction of aesthetic attitude in other natural resource environment, the whole set of variables of measures should be included (objective factors: income, visiting frequency, age, and educational level; subjective factors: purpose, reason, and sensation).

The aesthetic differences in Yan'an urban forests have certain representativeness because the mandatory occupational requirement further results in associated sightseeing and touring. In the study area, local government regularly organizes political and administrative education, resulting in the mixture of cultural landscape in urban forests. Previously, forestry development in Yan'an experienced a negative stage. The majority of residents had negative attitudes toward urban forestry and showed indifference to urban forestry construction and protection. Some residents were even involved in damage to urban forestry. In recent years, urban forestry has undergone rapid development in Yan'an. This change is certainly related to substantial improvement of economic conditions and the needs ${ }^{\circ}$ of residents for production and living; however, it is more dependent on the decision of the govermment in a stronger position.

In this study, we assessed the aesthetic value of urban forests in Yan'an and the individual differences in aesthetic altitude of visitors by taking into consideration of the unique ecoenvironment on the Loess Plateau in northern Shaanxi Prodince, as well as local eco-social conditions. The results of heretical assessment are generally consistent with the reality in the study area [16], thus providing a useful reference for urban forest operation and management.

However, assessment for aesthetic yalue of urban forests on the Loess Plateau is still in an early stage compared with aesthetic valuation of other resources with immature theories and methods [19]. There remain a number of issues that need to be further studied and solved in this developing and evolving field. Additionally, there are great differences among cities in terms of nature, society, and conomy rogether with different urban forest functions and services require a proper assessment index system for assessing the aesthetics of urban forests. It is recommended to combine environmental features and focus on the indicators such as the purpose and reason of local and foreigh visitors for visiting urban forests.

According to our results, errban forest functional status and cultural expression influence the direction and activity of the government and publics for investment on urban forestry. For sustainable developmen of urban forests, urban forest construction, management, and maintenance shall be taken as an economic behavior of government investment. While improving government competence, the associated economic rationality, residents' willingness to input, and the inheritance of urban culture should be emphasized. This is especially important in economically underdeveloped areas and circumstances where the economi gane of forests has not yet fully perceived by society. Application of the proposed method for assessing the aesthetic value of urban forests will help with management input and sustainable development of urban forests.

\section{Acknowledgements}

This paper is a revised and expanded version of a paper entitled "Assessing the aesthetic value of urban forests and asso-ciated individual differences: An example from the Loess Plateau, China" presented at SoftTech 2014, Yeosu, Korea, May 8-10, 2014. This study was supported by the National Forestry Public Sector Research Project of Science and Technology Department, National Forestry Bureau of China (201104045). It was also supported by the National Natural Science Foundation of China (61402234) and by the China Meteorological Administration grants (2014 MC 16, M43). Prof. Dexiang Wang is the corresponding author. 


\section{References}

[1] F. Di, Z. P. Yang, X. L. Liu, J. R. Wu and Z. G. Ma, "Estimation on aesthetic value of tourist landscapes in a natural heritage site: Kanas National Nature Reserve", Xinjiang, China. Chinese Geogr Sci, vol. 20, (2010).

[2] J. N. Martin, "The lover of the beautiful and the good: Platonic foundations of aesthetic and moral value. Synthese, vol. 165, (2008).

[3] L. Tahvanainen, L. Tyrvainen, M. Ihalainen, N. Vuorela and O. Kolehmainen, "Forest management and public perceptions - visual versus verbal information", Landscape Urban Plan,vol. 53, (2001).

[4] L.Tyrvainen, "Economic valuation of urban forest benefits in Finland", J Environ Manage, vol. 62, (2001).

[5] C. C. Harris, B. L. Driver ad W. J. McLaughlin, Improving the contingent valuation method: A psychological perspective, J Environ Manage, vol. 17, (1989).

[6] I. Ajzen and B. L. Driver, "Contingent value measurement: on the nature and meaning of willingness to pay", J Consum Psychol, vol. 1, (1992).

[7] E. Heyman, "Analysing recreational values and management effects in an urban forest with the visitoremployed photography method", Urban for Urban Gree, vol. 11, (2012).

[8] C. M. Bishop, Training with noise is equivalent to tikhonov regularizatjon, Neural Computation, vol. 7, no. 1 , (1995).

[9] G. W. Gross, J. M. Boone and D. M. Bishop, "Pediatric skeletah age - determination with neural networks", Radiology, vol. 195, no. 3, (1995).

[10] E. S. Rosenberger, M. D. Needham, A. T. Morzillo, and C. Mpehrke, "Áesthetical Attitudes, willingness to pay, and stated values for recreation use fees at an urban proximate forest, JForest Econ, vol. 18, (2012).

[11] N. H. Lansford and L. L. Jones, "Recreational and aesthetic value of watey using hedonic price analysis", J Agr Resour Econ, vol. 20, (1995).

[12] J. Meyerhoff, "Stated willingness to pay as hypothetical behaviour:can attitudes tell us more?", J Environ Plan Manage, vol. 49, (2006).

[13] K. Bernath and A. Roschewitz, "Recreational benefits of urbanforests: explaining visitors' willingness to pay in the context of the theory of planned behayior", J Environ Manage, vol. 89, (2008).

[14] D. Whittaker, J. J. Vaske and M. J. Manfredo, "Specificity and the cognitive hierarchy: value orientations and the acceptability of urban wildlife managementactions", Soc Natur Resour, vol. 19, (2006).

[15] E. Pouta and M. Rekola, "The theory of planned behavior in predicting willingness to pay for abatement of forest regeneration", Soc Natur-Resour, vol., 14, (2001).

[16] B. W. Kang, J. J. Liâ, L. Høu and L. M. Hán, "Health assessment of urban forest in Yan'an City", J Northwest A\&F Univ (Nat. Sci. Ed.), yó. 34 (in Chinese), (2006).

[17] J. Meyerhoff, "Stated wilkingness to pay as hypothetical behaviour: can attitudes tell us more? J Environ Plan Manage", vol. 49, (2006).

[18] E. S. Rosenberger. M. D. Needham, A. T. Morzillo and C. Moehrke, "Aesthetical Attitudes, willingness to pay", and stated values for recreation use fees at an urban proximate forest, J Forest Econ, vol. 18, (2012).

[19] Y. P. Wang, Y. X. He and L. Lian, "Analysis of Aesthetic Value and Tourism Development of Folk Custom Resources in Southeast Chong qing”, J Anhui Agri Sci, vol. 40, (in Chinese) (2012).

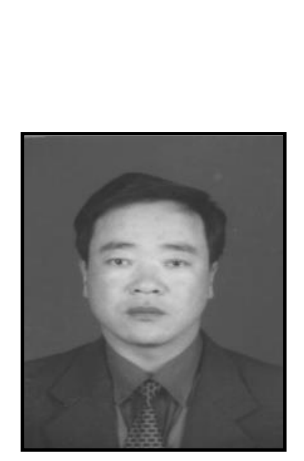

\section{Authors}

Dexiang Wang, he received his bachelor degree in agronomy from Northwest Forestry Collage in 1986, Ph.D. degrees in Agriculture Resources and Region Development from Northwest A\&F University in 2001. He is a professor in Northwest A\&F University in Shaanxi. His research interests include forest ecology, ecology management, and region development.

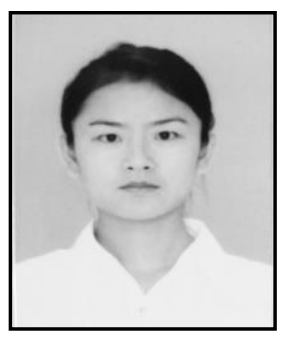

Mingxia Zhang, She obtained her bachelor degree in landscape architecture from Henan Agriculture University in 2005, M.S. degree in sivilculture form Northwest A\&F University in 2010. Now, she is working toward the Ph.D. degree in the Forest ecology. His current research interests are in quality assessment for urban forests. 